\title{
Accès au Logement Locatif au Cameroun : La Satisfaction d'un Droit Fondamental à L'épreuve des Contraintes Relationnelles avec les Propriétaires
}

\author{
Géneviève Noël Pieuwen Ngueukam, \\ Ismailla Datidjo, \\ Freddy Tsopfack Fofack, \\ Université de Dschang, Cameroun
}

Doi:10.19044/esj.2019.v15n28p213 URL:http://dx.doi.org/10.19044/esj.2019.v15n28p213

\section{Résumé}

Le droit au logement est généralement considéré comme un droit fondamental qui conditionne la satisfaction d'autres droits vitaux. La situation du logement dans les villes camerounaises fait pourtant état d'un déficit tant quantitatif que qualitatif de maisons d'habitation qui rend difficile l'accès au logement. Dans ce contexte, quelles stratégies déploient les citadins pour accéder au logement locatif, un droit conditionnant d'autres droits vitaux ? Comment ces personnes procèdent-elles pour se maintenir dans un appartement locatif en contexte de crise avérée du logement ? Ainsi, en enrégimentant la théorie de l'individualisme méthodologique pour analyser les entretiens menés avec les bailleurs, locataires et agents immobiliers dans les villes de Douala, Yaoundé, Garoua et Dschang, cet article rend intelligible les stratégies résidentielles face à la crise du logement au Cameroun. Au demeurant, habiter la ville du Cameroun prédispose le citadin locataire, à faire face aux contraintes lors de la demande, de l'acquisition et pendant le séjour dans un logement locatif. De même, être bailleur dans la ville camerounaise c'est être confronté dans la plupart des cas aux difficultés relationnelles avec son locataire. Dans la relation bailleurs-locataires, les acteurs impliqués rivalisent de stratégies pour tirer chacun de son côté le maximum d'avantages. À cet effet, le demandeur de logement, l'agent immobilier et le propriétaire rentrent dans un jeu d'intérêt qui rend davantage ardue la satisfaction du droit au logement en milieu urbain camerounais.

Mots clés : Logement locatif, Stratégies résidentielles, Crise du logement, Contraintes relationnelles, Demandeurs de logement, Bailleur. 


\title{
Access to Rental Housing in Cameroon: A Fundamental Right Satisfaction Fronting Constraints of Relationship with Proprietors
}

\author{
Géneviève Noël Pieuwen Ngueukam, \\ Ismaïla Datidjo, \\ Freddy Tsopfack Fofack, \\ Université de Dschang, Cameroun
}

\begin{abstract}
The right to housing is generally regarded as a fundamental right which determines the satisfaction of other vital rights. The state of housing in Cameroonian cities however indicates a quantitative and qualitative deficit of residential houses which makes access to housing difficult. In this context, what strategies do urban dwellers use to access rental housing, a right conditioning other vital rights? How do these people proceed to stay in a rental apartment in the context of a proven housing crisis? Thus, by using the theory of methodological individualism to analyze interviews conducted with landlords, tenants and real estate agents in the cities of Douala, Yaoundé, Garoua and Dschang, this article makes understandable the residential strategies of acquiring houses that are in front of habitation crisis in Cameroon. Moreover, living in a city in Cameroon predisposes the city dweller, to face constrains during the demand, the acquisition and the period of stay in a rented house. In the same way, being a house-owner in a town in Cameroon means to face relationship difficulties with the tenant even though it is not always the case. In the relation between the claimant of a rental house and the houseowner, the implicated actors challenge each other in strategies so as to obtain the maximum advantages. Due to this reason, the house seeker, the real estate agent and the owner enter into an interest game that makes more difficult the satisfaction of the right to housing in cities of Cameroon.
\end{abstract}

Keywords: Rental housing, Residential strategy, Habitation crisis, Relationship constrains, House seeker, Landlord

\section{Introduction}

Chaque individu a le droit de se loger décemment comme le stipule ONU-Habitat, dans son rapport de 2007. À en croire Boyer (2010) et 
Emmanuelli (2003), le logement rentre dans l'ordre des besoins élémentaires. Il conditionne l'accès à d'autres droits fondamentaux, notamment le droit à la santé, à l'éducation, au travail, à la vie en famille et à la vie privée etc. Le Cameroun a d'ailleurs ratifié divers instruments en faveur du droit au logement suffisant et convenable à l'instar de la Déclaration Universelle des Droits de l'Homme, du Pacte International sur les Droits Économiques, Sociaux et Culturels et la Déclaration de Vancouver, pour ne citer que ceuxlà. Au niveau national, la constitution du Cameroun dans son préambule protège le droit à la propriété qui est un élément du droit au logement. Toutefois, malgré les politiques de développement urbain et de l'habitat, la réalité rend compte du déficit notoire tant qualitatif que quantitatif du logement. Le Ministère de l'Habitat et du Développement Urbain (MINHDU) estime à un million cinq cent mille unités le déficit à combler en matière d'habitations ${ }^{4}$. Le Rapport National du Cameroun pour l'Habitat (2015) révèle par ailleurs que la construction des logis par les structures de promotion de la production des logements, notamment la Mission d'Aménagement et d'Équipement des Terrains Urbains et Ruraux (MAETUR), la Société Immobilière du Cameroun (SIC), la Mission de Promotion des Matériaux Locaux (MIPROMALO) ainsi que les promoteurs immobiliers privés, ne parviennent pas toujours à satisfaire la demande. Le gouvernement camerounais dans ses mesures de planification urbaine a inscrit un programme de dix-sept milles constructions de logements sociaux et d'aménagement de cinquante milles parcelles constructibles, comme le précise le DSCE (2009 : 62). Ce projet ainsi envisagé, devait être implémenté dans les grandes métropoles que sont Yaoundé et Douala, de même que dans les chefs-lieux de Régions et de Départements, y compris dans les villes universitaires, à l'horizon 2020. Pourtant, il est loin de parvenir aux objectifs escomptés. L'exécution de la composante habitat du Plan d'urgence triennal pour l'accélération de la croissance économique étalé de 2015 à 2017 n'est d'ailleurs pas parvenus aux objectifs escomptés, notamment la réalisation de 800 logements sociaux et des équipements collectifs associés dans les Chefslieux de Région hormis Yaoundé et Douala.

Les villes camerounaises souffrent donc d'une insuffisance de logements capables de combler les attentes des citadins. Cette situation est l'une des conséquences de l'évolution en dents de scie du cadre macroéconomique national qui se répercute dans le domaine de l'habitat. Les villes camerounaises connaissent une organisation anarchique du secteur du logement et par conséquent, les habitations sont pour la plupart loin de respecter les normes du logement prévues par l'ex Ministère du Développement Urbain et de l'Habitat (MINDUH), devenu aujourd'hui

\footnotetext{
${ }^{4}$ www.minhdu.gov.cm, consulté le 30/05/2019 à 13h45
} 
MINHDU ${ }^{5}$. Entre autres, il s'observe dans les grandes agglomérations du Cameroun, des habitations non sécurisées et insalubres où l'accès à l'eau potable et à l'électricité est loin d'être un acquis ; ce qui revient à dire qu'il persiste un nombre élevé de personnes mal logées. Pourtant, le décret $\mathrm{n}^{\circ}$ 2008/0737/PM du 23 avril 2008 du MINDUH fixe les règles de sécurité, d'hygiène et d'assainissement en matière de construction. En ce qui concerne la sécurité dans les constructions à usage d'habitation, la structure et les matériaux sont choisis pour résister avec une marge de garantie convenable aux efforts et attaques qu'ils peuvent subir et pour présenter un degré suffisant de résistance au feu. Ils doivent en plus protéger contre l'humidité, la variation de température, les conditions atmosphériques et assurer un isolement sonore suffisant.

Dans un contexte où le déficit de logement va grandissant, la population camerounaise fait de plus en plus face au problème d'accès au logement locatif. D'une manière générale, trouver un logement de qualité conforme à ses besoins et à ses ressources représente pour nombre de familles en ville un véritable défi. Quelles stratégies déploient-elles pour accéder au logement locatif, un droit conditionnant d'autres droits vitaux ? Et comment font-elles pour le préserver dans un contexte de crise avérée du logement ?

Répondre à ces questions consiste à appréhender les stratégies mises en place, selon une approche progressive ; pour cela, il convient de prendre en compte deux étapes du processus : le moment de la quête et de l'accès au logement et celui du séjour associé aux contraintes relationnelles entre bailleurs et locataires. Pour ce faire, cet article s'appuie sur les entretiens semidirectifs menés dans les villes de Douala, Yaoundé, Garoua et Dschang, avec cinquante-deux personnes, notamment douze bailleurs, huit agents immobiliers et trente-deux locataires de statuts sociaux variés. Notre choix de ces villes se justifie par leur caractère de ville régionale pour les trois premières, dont la capitale économique et celle politique du pays, et de ville Départementale et universitaire pour la dernière. Elles s'inscrivent dans les catégories de villes prioritaires en termes de construction de logements sociaux tel que prévue par les documents de planification à l'instar du Document de Stratégie pour la Croissance et l'Emploi (DSCE, 2009). En faisant recours à l'individualisme méthodologique de Boudon (1979) pour l'analyse des stratégies que développent les populations urbaines du Cameroun afin de satisfaire le droit au logement, ce travail rend compte des actions que construisent en permanence les individus en situation de déficit quantitatif et qualitatif du logement locatif.

${ }^{5}$ Le décret n²008/0737/PM du 23 avril 2008 du MINDUH, fixant les règles de sécurité, d'hygiène et d'assainissement en matière de construction disponible sur le site www.minhdu.gov.cm consulté le 06/12/16 à $17 \mathrm{~h} 00$. 


\section{Accéder au logement locatif au Cameroun : des stratégies diversifiées}

Dans les villes camerounaises, l'accès au logement locatif pour les nécessiteux est un exercice difficile et un défi majeur, compte tenu des conditions de vie précaires de très nombreuses familles et de l'insuffisance de logements pouvant les abriter. Ainsi, acquérir un logement locatif à la taille d'un studio ou d'un appartement, s'apparente à un véritable "parcours $d u$ combattant » car, il faut parfois chercher pendant longtemps pour se trouver un logis. Parler de stratégies résidentielles revient à restituer à l'acteur sa part d'initiative dans l'élaboration de sa propre existence (Godard, 1990). Nous entendons par là, l'effort que chaque individu déploie pour la satisfaction d'un besoin qui se pose à lui, précisément celui de se loger dans des conditions optimum. Les ménages, dans leur recherche de logement, déploient toute une série de stratégies et de compétences leur permettant d'accéder au bien recherché. Thomas et al. (2011) démontrent que les individus ont des compétences diversifiées par rapport à la recherche d'un logement et révèlent que la capacité à utiliser des outils de recherche de logements, tels que l'internet, les réseaux immobiliers et les annonces, constituent une stratégie d'accès au logement. Au Cameroun, la réalité est autre dans la mesure où les outils ci-dessus évoqués ne sont pas toujours privilégiés quand il s'agit de chercher à satisfaire le droit de se loger. Dans le cadre de leur quête d'habitations, les demandeurs dans les villes camerounaises développent diverses stratégies.

\subsection{La stratégie par connaissance arrimée à la méthode de «bouche à oreille "}

La stratégie par connaissance arrimée à la méthode de bouche à oreille est l'un des procédés les plus rependus pour accéder à un logement. Elle est fondée sur le capital social qui est fondamental dans l'acquisition du logis.

\subsubsection{Le capital social, un dispositif de possibilités pour obtenir un habitat locatif}

Le capital social est, selon Bourdieu (1979), le réseau de relations sociales que l'individu utilise dans un champ spécifique. Dans le contexte de quête d'un logis en ville, les camerounais interrogés mobilisent les connaissances directes ou indirectes à l'instar des membres de la famille, les amis et les collègues. Ces personnes qui sont disséminées à travers les coins de la ville, font recours à leurs réseaux interpersonnels, maximisant ainsi les chances de trouver plus vite un logement. Chaque maillon de la chaîne qui constituent le capital social du demandeur est ainsi tenu de recourir à son réseau de connaissances afin d'aider son proche qui se trouve dans le besoin. À cet effet, parce que le demandeur de logement est animé par la nécessité 
d'en trouver un, il ne se prive pas de rappeler au réseau ainsi constitué en sa faveur de ne pas perdre de vue sa doléance, ceci jusqu'à ce qu'il obtienne satisfaction.

\subsubsection{La rentabilisation du capital humain par les messages de bouche à oreille}

Lorsqu'un citadin est à la recherche d'un logement, il se constitue, sous son impulsion, un réseau d'informateurs qui propagent le message d'un individu à un autre. Pour reprendre un citadin rencontré à Yaoundé, lorsqu' « une personne a quelque chose sur la main », c'est-à-dire qu'elle est au courant de ce qu'une maison de location cherche un preneur, elle transmet l'information à la personne qui lui a émis le besoin. La diffusion du message de bouche à oreille consiste donc en une série de relais d'informations, dans un premier temps descendante, c'est-à-dire de la personne à la quête du logis aux personnes successives impliquées dans cette tâche, et dans un deuxième temps ascendante, parce qu'elle fait remonter l'issu de cette recherche. Le demandeur entre en contact avec le bailleur ou le concierge pour découvrir et faire l'état des lieux de cette maison et de s'enquérir des modalités pour y accéder. Au bout du compte, s'il a une perception positive de cette habitation, il remplit les conditions proposées et, au cas contraire, la recherche se poursuit en faisant élargir son réseau d'informateurs.

C'est pourquoi Thomas et al. (2011) stipulent que dans un contexte de pénurie, la stratégie de bouche à oreille permet de trouver la perle rare et d'être au courant de la future libération d'objets sur le marché locatif. Ainsi, au-delà de la pénurie de logements, certains ménages réduisent leur temps de recherche par la mobilisation de leur réseau de connaissances afin de trouver le logis qui leur convienne mieux. De ce fait, le capital social est le produit des stratégies d'investissement destinées à instituer ou à reproduire des réseaux de relations potentiellement profitables pour celui qui investit. Le rendement de ce type d'investissement est d'autant plus conséquent que le volume de capital détenu est important (Bourdieu, 1980). Dans la même perspective, Lallement (2006) affirme que le capital social est alors analysé non seulement comme les relations sociales souvent réduites à de purs échanges d'informations, mais aussi comme une ressource au profit des acteurs intéressés. Il ressort des propos de ces auteurs que, la rentabilité du capital humain c'est l'acquisition d'un ensemble de ressources par un acteur via ses relations. Ainsi dit, le capital social du demandeur de logement dans la ville camerounaise lui est rentable en ce sens où il lui permet d'acquérir un logement locatif sans lui valoir un investissement particulièrement considérable, tant en termes de temps à mettre personnellement en jeu qu'en termes d'effort à déployer. 


\subsection{La méthode par substitution de personnes et la stratégie de proche en proche}

Une autre stratégie déployée pour accéder à l'habitat locatif en milieu urbain camerounais est la méthode par substitution. Elle consiste, pour une personne déjà installée ou à la recherche d'un habitat, à intégrer une maison aussitôt qu'une autre personne directement ou indirectement connue la libère.

\subsubsection{La substitution négociée d'un locataire par un autre}

De prime abord, cette stratégie d'accès au logement voudrait qu'un individu intègre le logis que l'un de ses proches envisage de quitter. Pour cela, il le prévient de son intention de le substituer. En procédant ainsi, il garantit provisoirement l'appartement en attendant le départ du locataire en place. Lorsque ce dernier est prêt à déménager, il informe le bailleur et la personne pressentie pour y aménager.

Dans un second abord, cette stratégie voudrait que celui qui a manifesté le besoin d'accéder à la maison qui sera bientôt libérée, dispose des moyens financiers nécessaires pour assurer son occupation prochaine. Il ressort des entretiens menés que l'intégration du logis qui fait l'objet de convoitise se fait par la réservation préalable du locataire aspirant, tant auprès du locataire sortant que du propriétaire. À cet effet, un des informateurs à Garoua affirme :

La maison que j'habite présentement était d'abord habitée par un de mes collègues, il est allé aux États-Unis. Je lui avais demandé de me faire signe dès qu'il veut quitter la maison. Avant qu'il ne parte on est allé voir la bailleresse pour lui dire que quand il sort c'est moi qui entre et j'ai avancé l'argent.

Cette réservation se fait tantôt verbalement, tantôt moyennant une contrepartie. Dans la majorité des cas, le bailleur demande un dépôt de garanti qui excède le plus souvent trois mois de loyer. La stratégie par substitution arrimée à la stratégie de proche en proche milite pour le fait que, lorsque quelqu'un veut libérer une maison, il donne la priorité à une personne qui fait partie de son capital social de lui succéder. Eu égard à ce postulat, un locataire qui envisage sortir de sa maison de location informe ses pairs de cette opportunité d'accès au logement qui s'offre à eux sans qu'ils n'aient manifesté le besoin.

\subsubsection{L'obtention d'un logement locatif par personnes interposées}

Compte tenu de la rareté et de l'indisponibilité des logements locatifs au Cameroun, il est courant pour plusieurs informateurs d'acquérir une maison par le biais d'une personne qui s'apprête à déménager ou qui connaisse une autre personne dans cette situation. Ce transfert de logis d'un individu à un 
autre, repose généralement sur une connaissance directe, très souvent avérée entre un locataire sortant et celui qui envisage le remplacer. À titre illustratif, l'un de nos informateurs à Dschang affirme que l'accès au logement est une sorte de «(...) passation de service car, dit-il, il faut connaitre quelqu'un qui soit entrain de déménager, pour qu'il vous mette en contact avec le bailleur dans l'optique de vous faciliter l'obtention d'une maison, si le bailleur luimême n'a pas encore le remplaçant (...) ». C'est dans le même sens qu'évolue Bonvalet (1993) qui pense que la famille et les amis constituent une ressource importante dans la recherche de logement, car ils peuvent fournir des informations sur la disponibilité des habitats au demandeur. Toutefois, il advient que la personne en quête de maison parvienne à l'obtenir à partir d'une série d'intermédiaires dont la chaine de connaissance la mène à celle qui va bientôt déménager.

\subsubsection{Le tandem locataire-demandeur de logis : s'organiser et persuader le proprio}

Le troisième volet de la méthode par substitution arrimée à la stratégie de proche en proche se manifeste par les interactions entre le demandeur de logement et le locataire sortant, lesquels développent un jeu de stratégie qui ne laisse pas dans la plupart des cas la possibilité d'un choix alternatif au bailleur. À ce sujet, l'un de nos informateurs à Douala, au sujet du procédé d'accès à son logement, affirme que :

c'est quelque chose qui s'est passé à l'insu de plusieurs personnes y compris du bailleur ; c'est une sorte de permutation qui s'est opérée. Le monsieur qui sortait était le collègue d'un de mes amis, il a fait comprendre au bailleur que j'étais son frère et j'habitais déjà avec lui depuis longtemps. Il lui a donc dit que je vais juste continuer à y séjourner et que lui était sur le point de partir.

La stratégie par substitution interfère donc avec l'approche par connaissance dans les villes camerounaises comme à Genève en Suisse car, « trouver un appartement à Genève passe par des réseaux de relations » (Thomas et al., 2011 : 98).

\subsection{La location du logement à travers les agences immobilières}

Les agences immobilières sont des entreprises spécialisées dans les transactions immobilières. Elles assurent la médiation entre propriétaires et locataires ou entre vendeurs de biens immobiliers et acquéreurs. Elles reçoivent de la part de quelques propriétaires, les offres de logements locatifs et enregistrent les demandes des nécessiteux. 


\subsubsection{Le faible recours aux agences immobilières}

Peu sont les demandeurs de logement qui font recours aux agences immobilières. Ceci est lié au fait que la majorité des possesseurs de logements s'emploient eux-mêmes à trouver des preneurs pour leurs bâtisses, à travers des affiches dans les artères fréquentées de la ville et le recours aux relations sociales. Les propriétaires et les demandeurs sont tenus de verser à l'agence immobilière, d'une part, des frais d'enregistrement au départ et, d'autre part, un pourcentage sur la valeur du bien ou du loyer au cas où la médiation est concluante $\mathrm{Au}$ vu de ce qui précède, la majorité des bailleurs réussissent à faire louer leurs logis sans l'intervention d'un agent immobilier afin d'éviter des dépenses qu'ils considèrent incongrues.

\subsubsection{Les demandeurs de logis entre les propriétaires et les agents immobiliers}

Les locataires soulignent que les habitations qu'offrent les agents immobiliers coûtent plus cher que les logements de même standard directement négociés auprès des bailleurs. Ces derniers, bien qu'ils fixent les prix de loyer au moment de la mise à la disposition des agents immobiliers, ceux-ci les revalorisent dans le but de maximiser leur gain; les frais de commission de la médiation évoluant de façon proportionnelle au prix du loyer. En allant dans la même veine, Bonneval (2011) fait état de l'existence des logiques de marché dans le secteur immobilier qui, loin d'être exclusivement imputable aux particuliers, découlent du travail d'intermédiation des agents immobiliers. La stratégie d'acquisition d'un domicile locatif à travers les agences immobilières, voudrait que le demandeur de logement qui opte pour cette approche, entre en contact avec un agent immobilier. Celui-ci se charge de lui faire visiter, selon ses différents critères, les habitats locatifs disponibles parmi lesquels le demandeur de logement opère le choix.

\subsection{Le parcours des artères urbaines à la recherche d'un logement à louer}

Il est question ici d'un autre procédé pour avoir accès à un logement. L'individu motivé par le besoin d'acquérir un logement est appelé à faire permanemment des descentes sur le terrain afin de trouver quelque chose qui puisse le satisfaire. À cet effet, il parcourt les rues et ruelles de la ville sans cesse. Parfois, au bout de son effort quotidien, il n'obtient rien de satisfaisant, voire rien du tout. Pourtant, dans certaines circonstances, il trouve plusieurs opportunités d'acquisition d'une résidence. 


\subsubsection{L'exploration des affiches le long des routes}

Quand un individu s'engage à parcourir une ville au Cameroun à la recherche d'une habitation dans laquelle il pourrait aménager, son attention est le plus souvent focalisé sur les inscriptions publicitaires, sur les affiches qui jonchent les murs, les arbres et autres surfaces dans les rues. Sur ces affiches, il exploite toutes les informations indiquant l'existence d'un habitat locatif, pour aussitôt enregistrer l'adresse qui y est associée. Ainsi, il peut contacter la personne dont le numéro figure sur une annonce afin de se rapprocher de lui et de visiter le bâtiment pour une éventuelle négociation de son prix. Cette approche ressort clairement dans les propos de nos enquêtés, comme l'atteste précisément les dires de l'un d'eux :

(...) lorsque je cherchais la maison, j'ai trouvé une plaque sur laquelle étaient écrits maison à louer, deux chambres, un salon, une cuisine et une douche interne. Sur la même plaque il y avait aussi un numéro de téléphone ; celui de mon bailleur. Je me suis donc servi de ce numéro pour entrer en contact avec lui. C'est comme ça que j'ai fait pour trouver ma maison.

Ce témoignage illustre la contrainte à laquelle font face les chercheurs de logement qui sont parfois obligés de consentir d'efforts considérables pour se loger.

\subsubsection{La collecte d'informations auprès des habitants de quartiers}

Une autre option pour trouver un habitat au Cameroun en parcourant la ville, est de demander auprès des personnes rencontrées sur le chemin des renseignements sur l'existence éventuelle de maisons à louer dans leurs environs. Le nécessiteux est parfois orienté vers un propriétaire détenteur d'une habitation inoccupée. Selon les informations recueillies, ce procédé est fastidieux et nécessite un investissement considérable en temps et en effort, sans nécessairement déboucher sur le repérage et l'obtention d'un logement à louer.

Les stratégies d'accès à l'habitat locatif reposent sur des critères de choix eux aussi multiples du fait des exigences qu'elles imposent aux citadins nécessiteux.

\section{Les choix résidentiels : du désidérata à l'ambigüité}

Lorsque plusieurs choix se présentent à un demandeur de logement, dans chaque cas il examine les avantages et les inconvénients des possibilités qui s'offrent à lui et choisit celle qui lui est la plus favorable. Ainsi, le choix résidentiel puise dans les motivations qui poussent les citadins à porter leur inclination sur tel ou tel autre logis. 


\subsection{Les motivations du choix de la résidence}

Le choix résidentiel des locataires dans les villes camerounaises est déterminé par plusieurs facteurs :

La localisation de l'habitation par rapport aux lieux qui représentent les centres d'intérêt pour toute la famille est l'un des facteurs qu'évoquent les locataires. Goodman (1990), montre que le choix du logement précède le choix de la localisation. Contrairement à cette logique, au Cameroun, les demandeurs de logements sont plutôt attirés par la proximité des endroits tels que le centre-ville, la place de marché, les services publics, le lieu de travail et surtout les établissements scolaires où les enfants peuvent suivre leurs études etc. C'est d'ailleurs dans cette logique que Fack et Grenet (2009) pensent que les choix résidentiels des familles interagissent avec les choix scolaires entre autres, car l'instruction de leurs enfants est toujours une préoccupation pour les parents. La proximité de l'école devient pour cela un atout qui est pris en compte dans la recherche d'une maison à louer.

Compte tenu des contraintes liées à leurs revenus, les locataires voudraient limiter les dépenses qu'engendreraient l'éloignement des habitations de ces lieux ci-haut évoqués. Dans ce sens, l'avantage de la proximité des endroits régulièrement fréquentés par les populations, est de pouvoir faire ses courses à pied ou à moindre coût.

L'accessibilité à la route bitumée est un autre critère important pris en compte dans le choix de la localisation d'une maison. Bref, la principale préoccupation en ce qui concerne l'emplacement d'une habitation, c'est de limiter au maximum les dépenses qui en découleraient. À en croire Bonvalet et Dureau (2000), à Paris, de nombreux ménages modestes préfèrent les résidences locatives dans les quartiers centraux, même au prix d'une très forte promiscuité dans le logement. Au regard de ce que rapportent ces auteurs, il se dégage sans doute le fait que les mêmes motivations animent les familles modestes appelées à louer les habitations en zones urbaines.

Par ailleurs, les citadins locataires définissent et justifient leur choix résidentiel à partir du désir de la tranquillité, car disent-ils, les «endroits calmes », les «secteurs non bruyants » permettent de bien se reposer et de récupérer après une longue journée de travail ; ces endroits sont des cadres propices de révision pour les apprenants. De telles locutions sont utilisées à plusieurs occurrences et s'inscrivent dans la perspective de Thomas et al. (2011 : 53) qui affirme que "le calme est une dimension essentielle dans le choix du lieu de vie. ». L'accent porté sur ce critère dans la rationalité des acteurs laisse entendre qu'il y a un enjeu important autour du sentiment de sécurité, qui se rapproche probablement du rôle que joue l'environnement de vie dans la constitution de ce que Giddens et Meyer (1994) nomment la « sécurité ontologique », autrement dit, le type de sécurité qui est au fondement de la confiance en soi. 
De manière générale, les locataires dans les villes camerounaises portent leur choix sur des logis pour au moins une des raisons suivantes : la disponibilité du logement; l'avantage du nombre de pièces que renferme le logis ; la réputation et l'image favorable du quartier ; la proximité du lieu de travail et des écoles ; la proximité avec la route bitumée ; le coût abordable du loyer ; la disponibilité d'une source de provision continue en eau comme le puits ou le forage ; la facilité d'accès aux services tels que le transport en commun et les marchés entre autres. Bacqué et Vermeersch (2013) font aussi état des logiques multiples qui guident les choix résidentiels des classes moyennes en France. Ce sont les logiques familiale, professionnelle, économique et sociale auxquelles elles associent les «représentations que les citadins ont de l'espace urbain et des imaginaires dont il est le support » (p. 74). Au-delà de ces idéaux, nous soutenons l'idée de Berroir et al. (2016), selon laquelle «les choix résidentiels résultent d'un ajustement des aspirations subjectives des ménages à leurs contraintes objectives ».

\subsection{Les contraintes liées aux choix résidentiels}

Il est avéré que certaines résidences de location qu'offrent les villes camerounaises favorisent une stabilité résidentielle des locataires. Lorsqu'un citadin parvient à acquérir un logement qui lui est convenable, il le garde jusqu'à la fin de son séjour dans cette localité. C'est le cas pour cet informateur qui rapporte ceci : «je n'ai à envier aucun autre logement dans la ville de Douala, cette résidence renferme tout ce que j'ai toujours souhaité avoir dans une maison, je suis satisfait, il n'est pas question que je change de maison». À l'antipode de cette forme de stabilité résidentielle volontaire, apparait une autre qui est involontaire. Quelques-uns des informateurs révèlent qu'ils n'ont jamais changé de maison, non pas parce que la volonté n'y est pas, mais parce qu'ils n'ont pas trouvé une autre maison plus confortable que celle qu'ils habitent. Ils y sont par faute de trouver mieux, c'est ce qui se perçoit dans ce propos de l'un d'entre eux : «... si on quitte cette maison, où va-t-on aller? On n'a pas le choix, on s'adapte ». Une étude réalisée par Thomas et al. (2011) sur les choix résidentiels et les modes de vie, montre que la crise du logement oblige les ménages à de gros sacrifices ou encore à accepter n'importe quel logement. Ces propos permettent de comprendre qu'il s'agit d'une stabilité résidentielle contraignante.

Par ailleurs, une attention particulière est portée sur les nuisances physiques liées à l'existence par endroits des plantations, de la broussaille, des dépôts d'ordures à proximité des concessions; ce qui concoure à la prolifération des rats, des moustiques et d'autres bestioles qui peuvent en investissant les habitations, constituer des problèmes susceptibles d'affecter la 
santé des locataires. Selon l'OMS ${ }^{6}$, «La santé est un état de complet bien-être physique, mental et social et pas seulement l'absence de maladie ou d'infirmité ». Elle prend donc en compte les aspects environnementaux, sociaux, physiques etc. De ce fait, certains individus sont circonspects et hésitent parfois d'intégrer de tels logements qui ne leur garantiraient pas le droit à la santé.

À côté des contraintes liées à l'environnement physique et social, les maisons vétustes ou exigües et celles dont le nombre de pièces n'est pas en adéquation avec la taille du ménage ne sont pas toujours sollicitées. De même, les maisons situées dans les secteurs à nuisances sonores et à insécurité avérée ou supposée, n'attirent pas de demandeurs.

Certains bailleurs n'admettent dans leurs concessions que les demandeurs répondant à leurs critères de sélection. Ainsi, il se révèle que les individus qui disposent des salaires stables, bénéficient plus que toutes les autres, du privilège d'être admis dans une maison de location. Cependant, tout le monde ne jouit pas du même traitement de la part des proprios car, l'origine ethnique ou régionale du demandeur de logement peut lui valoir ou pas, la confiance du bailleur. Quelle que soit la ville où l'on se trouve, «avoir un nom à consonance autochtone accorde plus de facilité à obtenir un logis auprès de certains bailleurs autochtones ». Ce qui signifie qu'être originaire du terroir, offre plus d'avantages de trouver une résidence auprès des bailleurs ethnocentriques. De ce fait, la discrimination de certains locataires facilite l'accès au logement aux individus «autochtones» au détriment des «allogènes ».

Par ailleurs, d'autres bailleurs refusent systématiquement de louer leurs habitations aux célibataires car, ils craignent que ceux-ci soient des «irresponsables» et développent des attitudes et comportements jugés maladroits tant vis-à-vis du voisinage immédiat que de l'édifice qui leur est accordé. Il s'agit là d'une autre forme de discrimination qui puise dans la subjectivité des propriétaires.

Ces deux dernières considérations sont assimilables aux «discriminations systémiques» décrites par Bourgeois (2013) qui montre qu'au-delà de l'arsenal juridique et réglementaire qui encadre la sélection des candidats au parc locatif Habitations à Loyer Modéré (HLM) en France, les bailleurs sociaux disposent des marges de manœuvre qui leur permettent d'édicter des règles locales informelles, détournant ainsi certains usagers de ce bien.

Une fois le choix du logement effectué, le locataire aspirant fait la rencontre du bailleur en vue de s'accorder sur les modalités d'accommodation.

6 Constitution de l'Organisation Mondiale de la Santé, 1946 disponible sur le site www. who.int consulté le 16/07/17 à 00h 02mn. 


\section{Les stratégies d'accommodation à l'habitat et les relations locataires bailleurs}

Bien qu'affectée par la crise de l'habitat, les villes du Cameroun offrent tout de même aux demandeurs de logements, des studios et appartements. Mais le fait marquant est que, les habitations sont insuffisantes, presque jamais disponibles et ne correspondent pas toujours à leurs appétences. Compte tenu de cette situation, les demandeurs de logements sont obligés de développer des stratégies afin d'avoir accès à un logement qui corresponde à leurs aspirations, lesquelles stratégies interpellent inévitablement une interaction entre ces derniers et les bailleurs. Il s'agit ici de retracer les différentes stratégies qu'implémentent les locataires pour accommoder leur séjour aux modèles d'habitations qui se présente à eux ; en même temps, de donner un aperçu des relations qu'entretiennent ces locataires avec les proprios.

\subsection{Les procédés d'accommodation aux logements disponibles}

Les citadins locataires du Cameroun ne tarissent pas de stratégies lorsqu'il s'agit de rendre agréable leur cadre de vie. Autrement dit, les individus disposent au cours de leur séjour, d'une liberté d'action qui leur permet de contourner les difficultés pour en faire des atouts. À ce titre, Brun (1990) relève que les individus usent de leur esprit imaginatif pour mettre en œuvre des mesures favorables au séjour dans une habitation au préalable inconfortable. En outre, Bonvalet et Dureau (2000) relèvent que dans de nombreuses métropoles en France, la cohabitation intergénérationnelle constitue un moyen d'adaptation pour les ménages à la crise de logements. Pour renchérir cette idée, le courant individualiste soutient que les individus sont des acteurs innovants parce qu'ils développent sans cesse des stratégies tout à fait conscientes pour faire face aux réalités quotidiennes (Boudon, 1979). S'appuyant sur la grille d'analyse individualiste, Padiolo (1986), cité par Durant et Weil (1997) affirme que les individus engagés dans une situation dont les caractéristiques sont plus ou moins contraignantes poursuivent des buts en manipulant des ressources qui se traduisent en des comportements significatifs.

Au Cameroun, il nous a été donné d'inventorier quatre stratégies mises sur pied par les locataires pour faire face à la crise du logement. Cette dernière se comprend à travers l'agrégation des stratégies ou des actions que développe la population dans le but d'acquérir une habitation. Face à la pénurie de logements, les informateurs en tant qu'acteurs sociaux motivés, mettent en œuvre ces stratégies leur permettant de trouver satisfaction dans leur logement et de s'adapter ainsi au déficit qualitatif et quantitatif du logement que connaît le pays. 


\subsubsection{L'obtention d'un logement au prix des enchères élevées}

Motivés par le souci d'accéder à un logement qui leur convient, les demandeurs qui se succèdent auprès des bailleurs sont soumis à la concurrence du plus offrant. En effet, certains proprios retardent l'octroi de leurs maisons de location en refusant de servir les premiers venus, pour les faire rivaliser indirectement entre eux, à partir des prix proposés. Sous cet angle n'accède à un appartement disponible, que le meilleur offrant car, la réalité est que, la demande de logement est supérieure à l'offre.

\subsubsection{Le payement anticipé du prix de location des appartements en construction}

Dans le but d'accéder à un logement, certains demandeurs s'emploient à soutenir le financement des travaux à titre de payement anticipé des frais de bail des appartements en cours de construction. De ce fait, après avoir ciblé une résidence susceptible de combler ses attentes mais qui est inachevée, le demandeur de logement se propose d'accompagner financièrement la finition des travaux de construction afin d'y intégrer. Le montant que débourse ce demandeur pour booster l'achèvement des travaux, varie entre la somme d'argent équivalant à six mois de bail au moins et celle qui correspond à deux ans de loyer au plus. Cette stratégie présente pour l'aspirant locataire un double avantage : d'une part, il s'agit de garantir la maison et d'autre part, d'avancer considérablement les frais de location.

\subsubsection{La réhabilitation des habitations par le demandeur de logement}

Certains locataires affirment qu'ils résident dans des logements qu'ils ont réhabilités ; il s'agit d'anciennes habitations et des logis à équipements défectueux. Ce sont des concessions caractérisées notamment par l'insalubrité liée à la vétusté perceptible à travers le sol et les murs fissurés, les portes et les fenêtres faites de bois non massif et qui sont en délabrement, les marches détériorées etc. La réfection d'une telle habitation se fait avec l'accord du propriétaire, par le demandeur de logement ou le locataire.

De prime abord, les frais des travaux concernant la réhabilitation du logement, sont assumés uniquement par le bailleur avant de faire intégrer l'aspirant locataire.

Dans le deuxième abord, les charges sont partagées entre le proprio et le locataire, car les retouches relèvent exclusivement des souhaits de ce dernier qui y loge déjà, sans nécessairement être un impératif aux yeux du propriétaire.

D'après le troisième abord, le coût de la réfection est supporté uniquement par la personne qui aspire à occuper un logement ou qui l'occupent déjà. Dans ce cas, les aspirants locataires sont dans une position de faiblesse face aux propriétaires lorsqu'il doit y avoir négociation et qu'à 
certains égards, le propriétaire jouit d'une position dominante sur les demandeurs de logement (Thibodeau, 2003).

Compte tenu de la rareté des maisons, certains informateurs révèlent qu'ils ont dû réhabiliter leur logement à leurs propres frais avant d'y accéder. Certains tenants de logis locatifs au Cameroun qui disposent de vieux appartements malpropres, les mettent en location nonobstant cet état. Il est à noter que la condition d'accès qu'ils proposent aux aspirants locataires est, soit d'intégrer ces habitats tels qu'ils se présentent, soit de les réfectionner eux-mêmes.

\subsubsection{La mobilité résidentielle}

La mobilité a toujours fait partie du mode de vie des populations. Elle est considérée en général comme une stratégie de lutte contre la vulnérabilité existentielle. Rossi (1955) va dans le même sens lorsqu'il démontre que la fonction principale de la mobilité c'est d'ajuster le logement d'une famille à ses besoins. Par similitude, la mobilité résidentielle peut être considérée au Cameroun comme une stratégie d'amélioration des conditions de logement. Face à la situation de crise que connait le secteur de logement au Cameroun, il arrive que le demandeur accède d'abord à un logis provisoire. Il s'agit du premier logis «de passage » qu'il occupe pendant une certaine période, ou pendant le temps qu'il lui faudra pour trouver une autre maison qui corresponde à ses exigences. La majorité des personnes interviewées ont déjà connu la mobilité résidentielle au moins une fois. Elles sont pour cela motivées par la volonté d'adapter leur habitation non seulement à leurs exigences, mais aussi à leurs revenus. Les raisons ci-après sont évoquées par les informateurs pour justifier le changement de résidence :

- l'inadéquation entre le nombre de pièces et la taille du ménage, en raison de l'encombrement de la maison par les personnes et leurs ustensiles. Dans cet ordre d'idée, Agbossou (2007) souligne que la modification de la taille du ménage est une des fréquentes raisons de mobilité résidentielle ;

- la quête d'une autonomie résidentielle ou de l'émancipation résidentielle, c'est-à-dire quitter du statut d'hébergé ou de colocataire, pour le statut de locataire ;

- la quête d'une résidence luxurieuse et assez spacieuse qui correspondrait à son pouvoir d'achat, à la taille de son ménage, à la quantité de biens de consommation à disposition ou que l'habitant ambitionne en disposer car, les petits logements retiennent moins longtemps leurs occupants que les grands logements (Levy et Piazzoni, 1994) ;

- le souci de se rapprocher du lieu de travail ; 
- la quête d'un environnement sécurisé avec une bonne réputation. Cette raison témoigne de l'importance que les individus interrogés accordent au fait de vivre dans un environnement sûr comme le stipulent Bonvalet et Dureau (2000) ;

- la recherche d'un environnement « haut de gamme » favorisant un mode de vie individualiste pour chaque ménage. Cette posture mesurerait donc la volonté de «distinction sociale » selon les termes de Bourdieu (1979). Les informateurs mettent l'accent sur l'accessibilité des automobiles, que sur la réputation du quartier, l'alimentation permanente en eau, la proximité des services, pour caractériser un tel environnement.

Les raisons ci-dessus évoquées par les individus interrogés justifient leurs attentes en matière d'habitat. Elles se résument par les propos de Driant et al. (2005) qui pensent que la majorité des déménagements est motivée par des problèmes de qualité propres au logement et à l'adéquation à la situation familiale. Les changements de logement sont donc essentiellement liés au désir d'adapter son confort à la taille de sa famille, de changer d'environnement et de lieu de résidence. La mobilité résidentielle est à cet effet motivée par des questions propres au logement et/ou à la famille. Il ne faut pas perdre de vue que la mobilité résidentielle dans les villes du Cameroun dépend aussi des relations qui existent entre bailleurs et locataires. Le comportement de l'un vis-à-vis de l'autre est susceptible de causer des désagréments ou des effets pervers capables de provoquer le changement de domicile.

\subsection{Les relations locataires-bailleurs}

De manière générale, il existe deux types de relations entre propriétaires et locataires. L'une est conflictuelle alors que l'autre est fondée sur la camaraderie ou sur l'entente. Pour convenir avec Boudon (1979), nous entrevoyons le résultat d'actions réciproques des individus car, elles s'attachent des significations subjectives interprétables par les acteurs ; ce qui leur permet de tenir compte du comportement des autres et éventuellement de modifier le leur.

\subsubsection{Les conflits entre propriétaires et locataires}

Face à certaines situations, les bailleurs et les locataires ne parviennent pas à trouver un terrain d'entente. C'est ce que Boudon (1977) appelle « effets pervers », qui désignent dans notre contexte le conflit que les actions ou les comportements du bailleur ou du locataire sont susceptibles de générer. Cet auteur affirme qu' " il y a effet pervers lorsque deux individus (ou plus) en recherchant un objectif donné, engendrent un état de choses non recherché et 
qui peut être indésirable du point de vue soit de chacun des deux, soit de l'un des deux » (p. 20).

Les facteurs de conflit, c'est-à-dire de désaccord, entre les deux parties sont présentés ci-dessous.

\subsubsection{La majoration unilatérale du coût des loyers : une source de désaccord}

La révision d'une clause du contrat de bail, particulièrement le coût du loyer, tient compte de la densification de la demande. Les bailleurs prennent parfois l'initiative d'augmenter le montant du loyer mensuel, sous le prétexte que leurs appartements ne sont pas payés proportionnellement à leur valeur. Certains locataires cèdent à cette initiative alors que d'autres en sont réticents. En cas de désapprobation, certains bailleurs véreux et déterminés se servent de leurs droits de propriété pour abuser de la confiance des locataires qui collaborent sans aucune preuve matérielle des transactions qu'ils opèrent, afin de les mettre au pas ou de les amener à déménager. Un informateur de Yaoundé affirme :

J'ai les ennuis avec ma bailleresse, elle veut augmenter le prix du loyer, ce qui ne m'arrange pas, elle m'a menacé en disant qu'elle devait rencontrer un huissier de justice pour me faire partir soit disant que je lui dois des mois de loyer impayés, pourtant ce n'est pas le cas. Ce qu'il m'a incriminé, c'est le fait de n'avoir pas de preuve physique du payement de frais de mon loyer pour me défendre d'une éventuelle procédure pénale abusive.

Au regard du passage qui précède, il se cache une stratégie subtile que déploie le proprio pour influencer son locataire en faisant valoir son «pouvoir» de bailleur dans un contexte de supériorité de la demande résidentielle par rapport à l'offre. Ils évoquent pour ce faire l'alinéa 1 , de l'article 322 du Nouveau Code Pénal en vigueur au Cameroun depuis juillet 2016 qui pénalise les filouteries de loyers.

Bien plus, les informateurs relèvent qu'en cas de nécessité de réhabilitation du logis, il y a des bailleurs qui refusent de supporter la charge. Dans ce cas, il revient au locataire de réaménager le domicile à ses propres frais. Par contre, lorsque la charge des travaux est portée par le propriétaire, il profite de cette occasion pour revoir à la hausse le coût du loyer.

\subsubsection{La transgression des termes du contrat par le locataire ou par le bailleur}

Le Non-respect de certaines clauses du contrat, en l'occurrence le retard de payement de loyer, est une autre source de conflit entre bailleurs et locataires. Le constat est que le non-respect des termes du contrat par l'une des parties, crispe les relations locataires-bailleurs, ce qui dans certains cas, se 
manifeste par le harcèlement du premier par le second. Le harcèlement désigne le comportement répétitif et indésirable qui porte atteinte à la dignité de la personne tout en créant une atmosphère menaçante, hostile, méprisable, vexant ou offensant. Dans la même situation, d'autres bailleurs prennent pour résolution d'expulser les locataires de leurs propriétés en refusant par exemple de renouveler le contrat de bail.

La réhabilitation par les locataires des habitations inhospitalières parce que vétustes ou en état de délabrement est une source de différends. Il existe au Cameroun, des bailleurs mal intentionnés qui laissent des locataires réfectionner leur logement locatif et par la suite, s'arrangent à les mettre à la porte. D'autres font plutôt des remontrances et donnent des directives aux locataires, sans tenir compte du fait que le contrat de bail et le payement des frais du loyer donnent le droit au locataire de disposer de la maison à la limite des clauses qui régissent l'accord entre lui et le propriétaire.

\subsection{La relation de bonne intelligence entre le locataire et le bailleur}

La relation de familiarité entre bailleurs et locataires se traduit par l'attitude positive de l'un vis-à-vis de l'autre, il s'agit d'une forme d'entente et d'amitié qui se développe entre ces derniers. Pour qu'une relation de familiarité existe entre un locataire et son bailleur, chacun d'eux est tenu de respecter les termes du contrat de bail qui porte entre autres sur le payement régulier des coûts du loyer et l'entretien de l'infrastructure. Dans ce cas, il va de soi que le climat de convivialité prévale et ne laisse pas de place à la rancœur. Avant d'intégrer un logement locatif, le demandeur et l'offrant s'accordent sur certains points destinés à réguler leurs comportements les uns envers les autres. Ceux-là varient d'un logement à un autre et en fonction du quartier d'implantation.

De manière générale, selon les entretiens menés, le locataire est astreint, entre autres, de s'acquitter de la caution initiale de location, de payer le loyer et toute autre somme due aux termes convenus dans les délais impartis, de rendre l'habitation au propriétaire dans un état viable et de ne pas transformer le logement loué sans accord écrit du bailleur. Le bailleur quant à lui s'engage à assurer au locataire la jouissance paisible du logement, à ne pas s'opposer aux aménagements réalisés par le locataire, dès lors que ceux-ci ne constituent pas une transformation du logement, ni une violation des termes de l'accord ; remettre gratuitement une facture de loyer au locataire qui en fait la demande.

\section{Conclusion}

Dans un contexte de crise du logement au Cameroun, les citadins déploient diverses stratégies pour avoir accès aux habitats locatifs. Afin de rendre moins ardue la recherche du logis, l'essentiel est fait par la chaîne 
d'individus qui constituent le capital social du demandeur, par l'aspirant locataire lui-même et par les agents immobiliers. Toutefois, les stratégies déployées ne débouchent pas impérativement à la trouvaille du logement correspondant aux idéaux résidentiels des demandeurs. Les difficultés d'accès au logement laissent émerger une diversité de contraintes auxquelles ils font face. Ainsi, les quêteurs de logement sont soumis à la stabilité résidentielle involontaire; à l'insécurité résidentielle liée à l'environnement physique et social des logis disponibles jugé parfois inadéquat et aux discriminations des propriétaires sur la base de certaines considérations socio ethniques. Quand il advient que le demandeur trouve un logement, il est dès lors en interaction permanente avec le bailleur au cours de l'accommodation et du maintien dans le logement.

L'accommodation du logement à ses appétences passe par des stratégies variées qui mettent le demandeur face à des contraintes financières, liées aux modalités d'intégration du logis, et résidentiels, à travers l'obtention d'un habitat «de passage». La préservation de ce bien précieux par le locataire dépend de la nature des relations qu'il entretient avec son bailleur. Sur le marché immobilier, lorsque les actions ou les comportements du bailleur et/ou du locataire génèrent des "effets pervers », le locataire est en position de faiblesse et peut éventuellement perdre son logis. Le bailleur est donc en situation de force ou de pouvoir, eu égard à la supériorité de la demande de logis par rapport à l'offre. Dans le cas où chacun respecte les termes du contrat, il règne entre les deux partis un climat de bonne intelligence qui traduit la positive attitude de l'un vis-à-vis de l'autre. Habiter la ville au Cameroun passe donc par un ensemble de stratégies et de contraintes, depuis l'étape de la recherche jusqu'à celle du maintien ou de la résidence, marquée par les contraintes relationnelles entre bailleurs et locataires, en passant par celle de l'accommodation.

\section{References:}

1. Agbossou, I. (2007). Modélisation et simulation multi agents de la dynamique urbaine : application à la mobilité résidentielle. Thèse présentée en vue de l'obtention du titre de docteur en géographie, Université de Franche Comté.

2. Bacqué, M.-H. et Vermeersch S. (2013). «Les classes moyennes dans l'espace urbain : choix résidentiels et pratiques urbaines. », Sociologie et sociétés, vol. 45, $\mathrm{n}^{\circ}$ 2, pp. 63-85.

3. Berroir, S., Delage, M., Fleury, A., Fol, S., Guerois, M. et al. (2016). "Trajectoires résidentielles, construction des espaces de vie et ancrage dans le périurbain. Enquête au nord de l'agglomération parisienne. », Revue française des affaires sociales, La documentation française, vol. 
3, $\mathrm{n}^{\circ}$ 7, pp.65-89. www.cairn.info/revue-francaise-des-affairessociales-2016-3-page-65.htm. hal-01391425.

4. Bonneval, L. (2011). Les agents immobiliers : Pour une sociologie des acteurs des marchés du logement. Nouvelle édition [en ligne]. Lyon, ENS Éditions, DOI : 10.4000/books.enseditions.4468.

5. Bonvalet, C. (1993). «Le transmis et l'acquis : localisation, statut d'occupation et type d'habitat ». In C. Bonvalet et A. Gotman (dir.), Le logement, une affaire de famille. Paris, L'Harmattan, pp. 23-40.

6. Bonvalet, C. et Dureau, F. (2000). «Les modes d'habiter: des choix sous contraintes ». In F. Dureau, V. Dupont, E. Lelièvre (dir.), Métropoles en mouvement. Paris, Anthropos, pp. 131-153.

7. Boudon, R. (1977). Effets pervers et ordre social. Paris, PUF.

8. Boudon, R. (1979). La logique du social, Paris, hachette.

9. Bourdieu, P. (1979). La distinction : critique sociale du jugement. Paris, Les Éditions de Minuit.

10. Bourdieu, P. (1980). «Le capital social ». Acte de recherche en sciences sociales, $\mathrm{n}^{\circ} 31$, pp. 2-3.

11. Bourgeois, M. (2013). «Choisir les locataires du parc social ? Une approche ethnographique de la gestion des HLM ». Sociologie du travail, vol. $55, \mathrm{n}^{\circ} 1$, pp. 56-75.

12. Boyer, F. (2010). «Croissance urbaine, statut migratoire et choix résidentiels des ouagalais. Vers une insertion urbaine ségrégée? », Revue Tiers Monde, vol. 1, $\mathrm{n}^{\circ}$ 201, pp. 47-64. https://www.cairn.info/revue-tiers-monde-2010-1-page-47.htm

13. Brun, J. (1990). « Mobilité résidentielle et stratégies de localisation ». In C. Bonvalet et A. M. Fribourg (dir.), Stratégies résidentielles. Paris, INED, pp. 299-312.

14. Constitution de l'Organisation Mondiale de la Santé, 1946 sur le site www.who.int consulté le 16/07/17.

15. Décret n²008/0737/PM du 23 avril 2008 du MINDUH fixant les règles de sécurité, d'hygiène et d'assainissement en matière de construction, www.minduh.gov.cm, consulté le 06/12/16.

16. Driant, J.-C., Drosso, F. et Vignal, C. (2005). Les Français et le logement : bouleversements de la démographie et de la famille. Quels choix résidentiels pour demain? Paris, CRETELL, Institut d'urbanisme de Paris.

17. Durant, J-P et Weil, R. (1997). Sociologie contemporaine. Paris, vigot, 2e édition.

18. Emmanuelli, X. (2003). Droit au logement : construire la responsabilité. 9e rapport du Haut comité pour le logement des personnes défavorisées, Paris. 
19. Fack, G. et Grenet, J. (2009). « Sectorisation des collèges et prix des logements à Paris ». Actes de la recherche en sciences sociales, vol. $180, \mathrm{n}^{\circ} 5$, pp. 44-62.

20. Giddens, A., et Meyer, O. (1994). Les conséquences de la modernité. Théorie sociale contemporaine. Paris, L'Harmattan.

21. Godard, F. (1990). « Sur le concept de stratégie ». In C. Bonvalet et A. M. Fribourg (dir.), Stratégies résidentielles. Paris, INED, pp. 9-22.

22. Goodman, A., C. (1990). « Demographics of Individual Housing Demand. » In J. Persky, Regional science and urban economics, n²0, pp. 83-102.

23. Lallement, M. (2006). «Capital social et théories sociologiques ». In A. Bevort et M. Lallement, Le capital social. Performance, équité et réciprocité. Paris, La découverte, pp. 71-88.

24. Levy, J-P. et Piazzoni, F. (1994). Qui habite quoi ? Composantes et dynamiques socio-spatiales du marché de l'habitat à Pantin. Rapport pour la ville de Pantin.

25. Nouveau Code Pénal du Cameroun, Juillet 2016.

26. ONU-Habitat. (2007). Global Report on Human Settlements: Enhancing Safety and Security.

27. Padiolo, J. G. (1986). L’ordre social. Paris, L'Harmattan.

28. Rapport National du Cameroun pour 1'Habitat. (2015). Troisième conférence des Nations Unies sur le logement et le développement durable en milieu urbain (Habitat III).

29. République du Cameroun. (2009). Document de Stratégies pour la Croissance et 1'Emploi, Yaoundé.

30. Rossi, P. H. (1955). Why families move? Beverly Hills, Sage Publications.

31. Thibodeau, J.-C. (2003). Évolution du marché du logement locatif : analyse, effets et perspectives. Société d'Habitation du Québec, Gouvernement du Québec.

32. Thomas, M-P., Adly, H., Pattaroni L., Kaufmann, V. et Galloux, S. (2011). Choix résidentiels et modes de vie dans l'agglomération franco-valdo-genevoise. Lausanne, Rapport final du mode de vie. 